\title{
Dynamics of an autumn phytoplankton bloom in a hypereutrophic urban lake in the semiarid Pampean region (Argentina)
}

Alberto Pilati, Santiago Andrés Echaniz, Alicia María Vignatti \& Graciela Inés Bazán

Facultad de Ciencias Exactas y Naturales, Universidad Nacional de La Pampa, Uruguay 151, 6300, Santa Rosa, La Pampa, Argentina.

\section{Correspondence}

A. Pilati

E-mail: apilatiunlpam@gmail.com

Received: 13 May 2019

Accepted: 12 February 2020

Published on-line: 26 March 2020

\section{Resumen}

Dinámica de un florecimiento algal otoñal en un lago hipereutrófico urbano de la región pampeana semiárida (Argentina).

En este trabajo, se reporta un raro florecimiento fitoplanctónico otoñal en un lago somero de la región semiárida pampeana ( $\mathrm{Ar}$ gentina). La biomasa fitoplanctónica presentó un solo pico otoñal, seguido dos meses más tarde por un pico en la clorofila. El pico de biomasa coincidió con un débil pastoreo sobre la comunidad fitoplanctónica (dominada por la poco pastoreada Planktothrix agardhii (Gomont) Anagnostidis \& Komárek) junto con una reducción de la limitación por $\mathrm{N}$ en el otoño. El pico de clorofila ocurrió cuando las algas aumentaron su contenido de clorofila probablemente debido a la reducción de la radiación solar y la baja transparencia del agua. Esto indicará que la estacionalidad del fitoplancton en esta área particular depende de múltiples factores bióticos y abióticos.

Palabras clave: Cambios fenológicos; Lago somero hipereutrófico; Planktothrix agardhii; Zooplancton; Limitación por N.

\begin{abstract}
In this paper we report a rare autumm phytoplankton bloom in a shallow lake of the semiarid Pampean area of Argentina. Phytoplankton biomass peaked only once during the autumn, followed 2 months later by a chlorophyll peak. The biomass peak might have been the result of a weak grazing pressure on the phytoplankton community (dominated by the nongrazeable Planktothrix agardhii (Gomont) Anagnostidis \& Komárek) coupled with a reduction in the $\mathrm{N}$-limitation towards the autumm. The chlorophyll peak took place when phytoplankton cells increased their chlorophyll content probably due to a reduction of solar irradiance and low water transparency. This findings indicate that phytoplankton seasonality in this particular area may depend on multiple biotic and abiotic factors.
\end{abstract}

Key words: Phenological shifts; Hypereutrophic shallow lake; Planktothrix agardhii; Zooplankton; N-limitation. 


\section{Introduction}

Succession in freshwater lakes has been extensively studied since the 80's. The Plankton Ecology Group (PEG) model (Sommer et al. 1986) predicted that eutrophic lakes have two phytoplankton blooms, one in spring and a more important one in summer. Those blooms, characterized by low water transparency, are separated by a clear water phase (high water transparency) caused by high zooplankton biomass and grazing. The applicability of the PEG model to shallow lakes or tropical lakes has not been very well documented (Lampert \& Sommer 2007). Nevertheless, Moustaka-Gouni et al. (2014) recently found that eutrophic Mediterranean lakes have a minimal phytoplankton bloom in spring followed by maximum biomass peak in autumn.

As the PEG model not always apply to all aquatic systems, many attempts have been made to determine the factors that affect phytoplankton peaks and their occurrence. Phytoplankton peaks can be measured as biovolume or chlorophyll as a surrogate of biomass. Biovolume calculation is time consuming and requires the expertise of the researcher in classifying the algae (Hillebrand et al. 1999). Chlorophyll measurements, on the other hand, can change with light, nutrient availability and phytoplankton composition, so caution is needed when using this surrogate as a biomass estimator (Kasprzak et al. 2008). Regarding occurrence, Winder \& Cloern (2010), using chlorophyll as a phytoplankton biomass estimator, described different aquatic systems with regular or irregular cycles which were affected not only by physical and chemical factors, but also by foodweb seasonality. They found that most aquatic systems show one peak in spring as a consequence of a temperature increase and water mixing with nutrient inputs. These authors also found that lakes with two peaks (spring and autumn or winter) were present in lakes with high grazing rates and shifts in the phytoplankton community, whereas lakes with irregular blooms generally respond to short-term climatic events which affect the lake's temperature and mixing dynamics. Other lakes, on the other hand, may show a shift in periodicity between the previous two patterns, or even the absence of a periodic pattern (generally hypertrophic lakes, with cyanobacteria domination all year long, and low grazing pressure by zooplankton) (Winder \& Cloern 2010) .
The Pampean region of Argentina, is characterized by the presence of a large number of shallow, eutrophic-hypertrophic, polymictic lakes (Quirós et al. 2002, Diovisalvi et al. 2015). Their phytoplankton communities are generally dominated by cyanobacteria which generally bloom during the warm season (Quirós et al. 2002, Izaguirre et al. 2015) as a result of water level fluctuations and eutrophication, or early spring depending on zooplankton grazing and light limitation (Torremorell et al. 2007). The western part of this region is located below the $700 \mathrm{~mm} /$ year isohyet (Diovisalvi et al. 2015) with mainly temporary, shallow, and saline lakes (Vignatti et al. 2017), with water level fluctuations depending on precipitation cycles (Viglizzo 2011). Some of these lakes had uncommon fall/winter chlorophyll peaks but no affecting factors were associated to them (Echaniz et al. 2013, Echaniz et al. 2016). To help understand factors driving the seasonality of phytoplankton in this area, we studied Don Tomás, a shallow urban lake with frequent summer chlorophyll peaks (Echaniz et al. 2008) that in 20082009 showed only one important chlorophyll and phytoplankton biomass peak (2-months apart) during the fall. Thus, we analyzed how nutrients, zooplankton and phytoplankton nutrient limitation may have triggered this rare phenomenon.

\section{Materials and methods}

\section{Study area}

Don Tomás lake is a hypereutrophic shallow lake (146 Ha) located by Santa Rosa, capital city of La Pampa province (Fig. 1) (36 $37^{\prime}$ S; 64 $4^{\circ} 19^{\prime}$ W). It is located on the eastern edge of the Thorny Forest ecoregion. The climate is semi-arid, with a temperature range between 8.9 and $23.6^{\circ} \mathrm{C}$, an annual mean temperature of $15.8{ }^{\circ} \mathrm{C}$. The average wind speed is around $13 \mathrm{~km} / \mathrm{h}$ and the annual rainfall is $746 \mathrm{~mm}$ (Morello et al., 2012). It is a region with gentle slopes produced by wind action, covered by open deciduous forest dominated by Prosopis caldenia Burkart, Prosopis flexuosa De Candolle, and Geoffroea decorticans (Gill. ex Hook. \& Arn.) Burkart (Morello et al., 2012). However, most of the basin has been currently deforested due to the increase in urbanization. Its waters are well oxygenated $(>6 \mathrm{mg} / \mathrm{L})$ and its ionic content characterized by the presence of sodium bicarbonate. Total suspended solids averaged $54.6 \mathrm{mg} / \mathrm{L}$ 


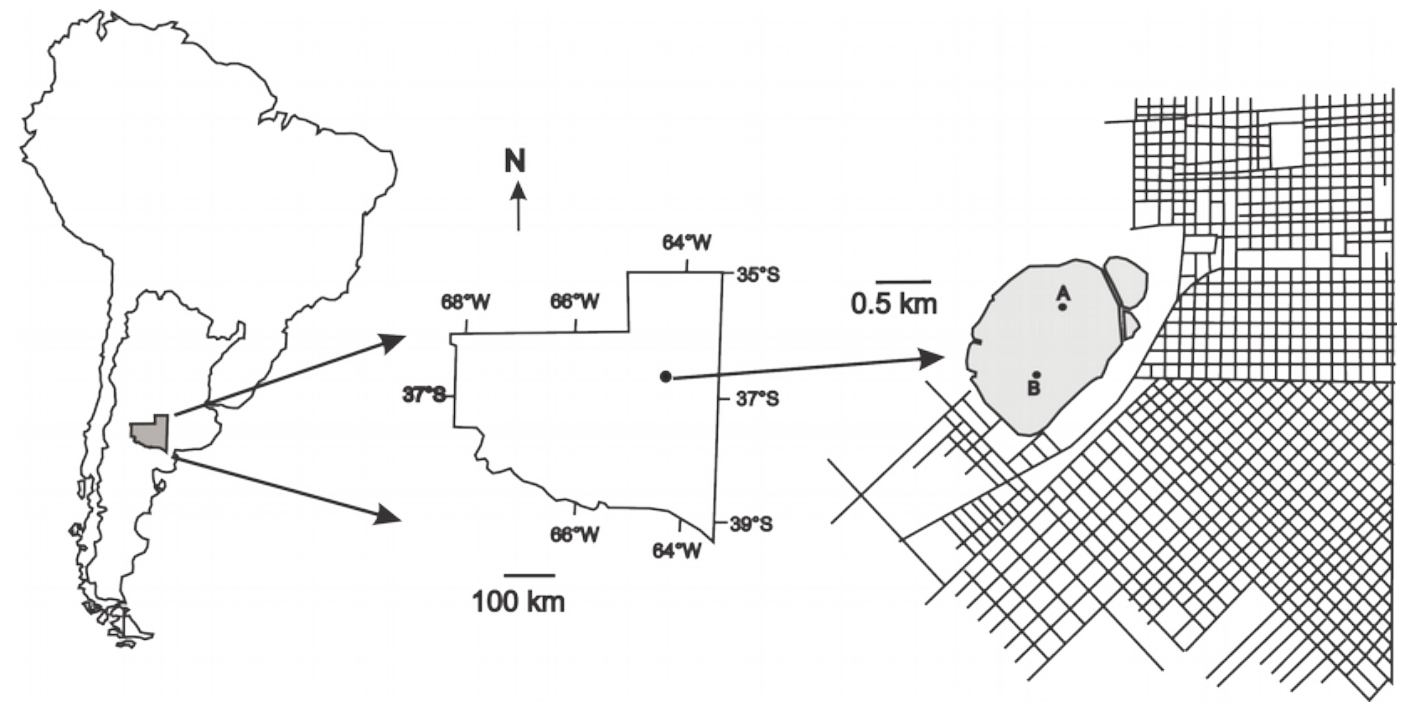

Figura 1. Mapa de la ubicación de la laguna Don Tomás (Provincia de La Pampa, Argentina), posición de los sitios de muestreo (A: 36 37' $11^{\prime}$ S, 6418'49"O y B: 36³7'29”S, 6418'56"O) y zona oeste de la ciudad de Santa Rosa.

Figure 1. Map showing the location of Don Tomás Lake (La Pampa province, Argentina), the position of the sampling sites (A: $36^{\circ} 37^{\prime} 11^{\text {' }} \mathrm{S}$, $64^{\circ} 18^{\prime} 49^{\prime \prime} \mathrm{W}$ and B: $36^{\circ} 37^{\prime} 29^{\prime \prime}$ S, 64'18'56'W) and the west side of Santa Rosa city.

and were mostly organic (Echaniz et al. 2008). From 1996 to 2006, as the city increased its drainage area, both the lake's salinity and water transparency decreased from 1.65 to $0.65 \mathrm{~g} / \mathrm{L}$ and from 0.24 to $0.14 \mathrm{~m}$ respectively (Echaniz et al. 2012). These authors suggest that water transparency reduction in this lake was associated with a decline in cladoceran grazers due to a zooplanktivorous fish, the silverside, Odontesthes bonariensis Valenciennes, 1835. In fact, silverside density has increased from $125.7 \mathrm{ind} / \mathrm{Ha}(25.5 \mathrm{Kg} /$ $\mathrm{Ha})$ in October 1998 to $220.9 \mathrm{ind} / \mathrm{Ha}(32.3 \mathrm{Kg} /$ $\mathrm{Ha}$ ) in June 2008 (O. Del Ponti, pers. comm.). Currently, the lake receives only the city storm drainage as the sewage drainage was diverted to other lake since 1987.

During this study, there was an important drought in the area, consequently Don Tomás Lake reduced its mean depth from $1.7 \mathrm{~m}$ at the beginning of the study to $1.5 \mathrm{~m}$ at the end. Lake temperatures ranged from $8{ }^{\circ} \mathrm{C}$ in winter (JuneJuly) to $26^{\circ} \mathrm{C}$ in summer, but the lake never stratified. Trophic state indexes based on water transparency, total phosphorus and chlorophyll (Carlson 1977) were always higher than $70(88.1 \pm 1.3$; $91.3 \pm 1.6$ and $75.9 \pm 5.4$ respectively) indicating the hypereutrophic condition.

\section{Sampling, analytical and statistical procedures}

From September 2008 to August 2009, we firstly determine the stratification regime by measuring monthly in situ temperature and dissolved oxygen profiles with a YSI oxygen meter (Model 55). These measurements were taken every $0.5 \mathrm{~m}$ at midday at two points $500 \mathrm{~m}$-apart in the deepest part of the lake (Fig. 1). As the lake was always well mixed (polymictic), monthly sub-surficial water samples $(0.10 \mathrm{~m})$ were considered representative of the water column at those sampling points. The study area, sampling protocols, water transparency and some laboratory analyses (chorophyll, and zooplankton biomass) are described elsewhere (Echaniz et al. 2012). Dissolved nutrients were analyzed after filtering the water through a GF/F glass fiber filter. Dissolved inorganic nitrogen (DiN) was considered to be the sum of only ammonia (phenate method; APHA 1992) and nitrate (cadmium reduction; Hach, method \#8039) as nitrite was below detection levels. Soluble reactive phosphorus (SRP) was measured with the ascorbic acid method (APHA 1992). Total nitrogen (TN) and phosphorus (TP) were digested with a peroxodisulfate oxidation following Ebina et al. (1983) and read with the cadmium reduction and ascorbic acid methods, respectively. All nutrients were measured with a Hach DR/4000 spectrophotometer.

Total suspended solids (TSS) were estimated by filtering lake water onto a pre-weighted Gelman $\mathrm{A} / \mathrm{E}$ glass fiber filter and drying the filter for 24 hours at $60{ }^{\circ} \mathrm{C}$. Organic suspended solids (OSS) were calculated by difference between TSS and non-volatile suspended solids (ashing the 
same filter (TSS) at 550C for 1 hour) (APHA 1992).

Phytoplankton samples were taken from a depth of $0.10 \mathrm{~m}$ and preserved with $4 \%$ formalin. Identification followed literature by Prescott (1951), Komárek \& Anagnostidis (1999, 2007), Komárek (2013), and Bourrelly (1985). Quantification was done using an inverted microscope Iroscope SH-24PH with a 10-mL Utermhöl settling chamber. Two hundred fields were counted. Phytoplankton biovolume was estimated by geometric approximation based on micrometer measurements of 25 randomly selected specimens (Hillebrand et al. 1999).

To quantify the severity of phytoplankton nutrient limitation, we took monthly sub-surficial water samples. Water was screened with a $63 \mu \mathrm{m}$ mesh to remove zooplankton and placed into $250 \mathrm{~mL}$ flasks ( 3 per treatment). There were four treatments: Control (no nutrients added), $+\mathrm{N}(150$ $\mu \mathrm{mol} / \mathrm{L} \mathrm{N}$ added as $\left.\mathrm{NH}_{4} \mathrm{NO}_{3}\right),+\mathrm{P}(9.3 \mu \mathrm{mol} / \mathrm{L} \mathrm{P}$ added as $\mathrm{Na}_{2} \mathrm{HPO}_{4} * 12 \mathrm{H}_{2} \mathrm{O}$ ) and $+\mathrm{N}+\mathrm{P}$. Flasks were incubated in an environmental chamber at $200 \mu \mathrm{mol} \mathrm{PAR} / \mathrm{m}^{2} \mathrm{~s}$ for $48 \mathrm{~h}$. We considered phytoplankton communities to be N-limited, P-limited, or N+P-limited when the chlorophyll level of the treatment (log-transformed) was significantly higher than the control using an one-way ANOVA followed by a Tukey test for treatment means comparisons. Percent of control responses were calculated following Marcarelli et al. (2006). To analyze the effect of light limitation on the chlorophyll content of algal cells, we used the $\mathrm{C}$ :Chlorophyll ratio. Although we did not measure $\mathrm{C}$ content in phytoplankton directly, we estimated it at $10 \%$ of algal biovolume (Peters \& Downing 1984).

Spearman's $R_{s}$ correlation coefficients were calculated to examine relationships among main limnological variables.

\section{Results}

In spring (September to December), chlorophyll had the lowest values $(\sim 50 \mu \mathrm{g} / \mathrm{L})$, while by late autumn (May and June) it had a distinctive peak $(\sim 191 \mu \mathrm{g} / \mathrm{L})$ (Fig. 2) almost 4 times higher than the spring values. Mean chlorophyll-a concentration was $115 \mu \mathrm{g} / \mathrm{L}( \pm 55.1)$. Water transparency was low $(0.14 \mathrm{~m} \pm 0.01)$. TSS (100\% organic) ranged from $45-60 \mathrm{mg} / \mathrm{L}$. Although water trans-

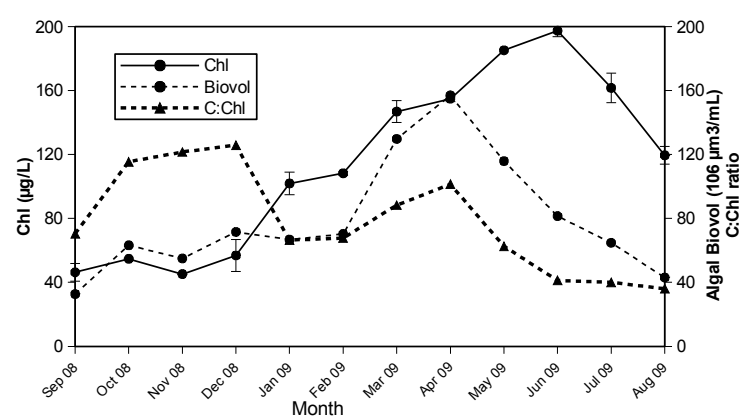

Figura 2. Concentración media mensual de clorofila (círculos. negros), biovolumen algal total (círculos blancos) y relación Carbono:clorofila (cruz) en la laguna Don Tomás, 2008-2009, Santa Rosa, La Pampa, Argentina.

Figure 2. Mean monthly chlorophyll concentrations (black circles), total algal biovolume (white circles) and $\mathrm{C}$ :Chl ratios (cross) in Laguna Don Tomás, 2008-2009, Santa Rosa, La Pampa, Argentina.

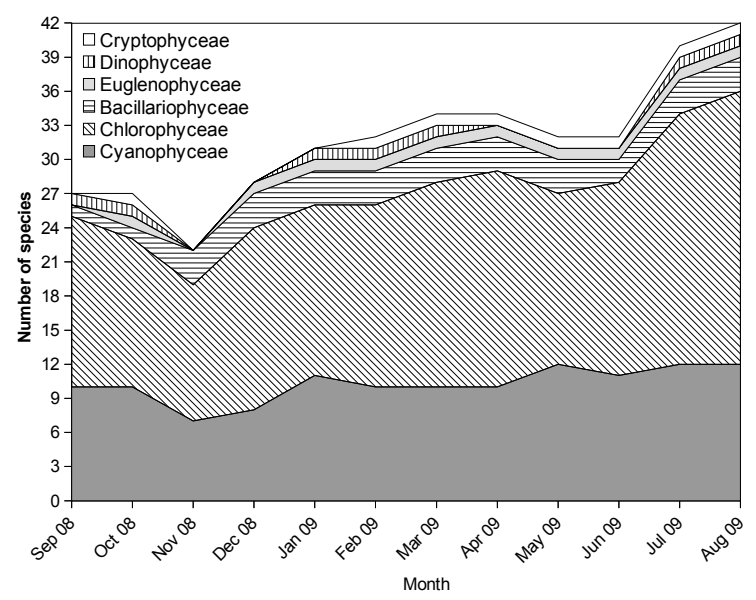

Figura 3. Riqueza de especies de grupos de fitoplancton en la laguna Don Tomás durante 2008-2009. La línea superior indica el número total de especies encontradas.

Figure 3. Species richness of phytoplankton groups in Laguna Don Tomás during 2008-2009. The top line in the figure indicates the total number of species found.

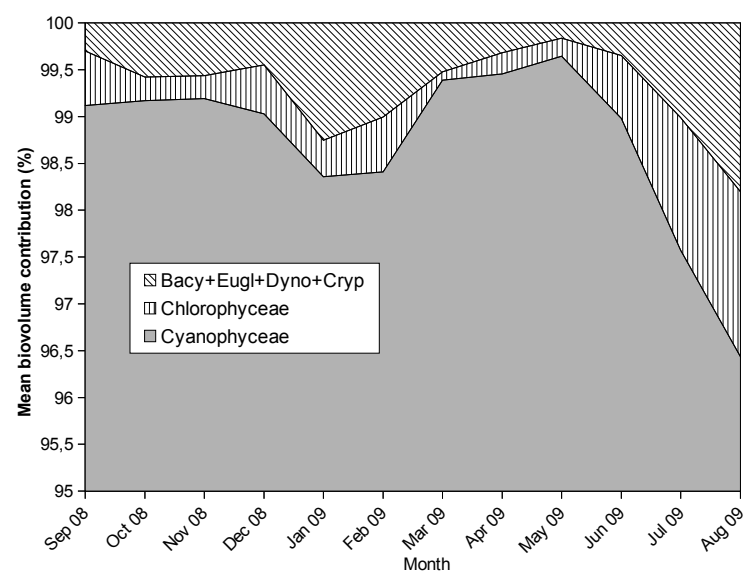

Figura 4. Contribución relativa del biovolumen (\%) de los principales grupos algales en la laguna Don Tomás durante 20082009.

Figure 4. Relative biovolume contribution (\%) of main phytoplankton groups in Laguna Don Tomás during 2008-2009. 
parency and TSS did not show any particular seasonal pattern, both were significantly and negatively correlated $\left(\mathrm{R}_{\mathrm{s}}=-0.952 ; \mathrm{p} \leq 0.0001\right)$. Chlorophyll, on the other hand, was not correlated with water transparency $\left(\mathrm{R}_{\mathrm{s}}=0.322 ; \mathrm{p}=0.291\right)$.

Mean algal biovolume during the study was $0.78 \mathrm{~mm} 3 / \mathrm{mL}( \pm 0.38)$, but in contrast to chlorophyll-a, it had a peak in mid-autumn (April) (Fig. 2). C:Chl ratios had a mean value of 78 , showing a minimum value of 36 in winter and a maximum value of 126 in spring (Fig. 2). Chlorophyll-a and algal biomass were significantly correlated $\left(\mathrm{R}_{\mathrm{s}}=\right.$ $0.664 ; p=0.018$ ).

Phytoplankton richness increased from 27 species at the beginning of spring (September 2008) to 42 species by the end of the winter (August 2009) (Fig. 3, Table 1). Chlorophyceae was the most diverse group (Fig. 3, Table 1). Nevertheless, Chlorophyceae had the lowest contribution to biomass in the phytoplankton community (Fig. 4). Cyanobacteria, on the other hand, never had more than 12 species but contributed more than $96 \%$ of total phytoplankton biomass (Fig. 4) with Planktothrix agardhii as the dominant species (Table 1). The dominant species during the biomass (April 2009) and chlorophyll (June 2009) peaks were Plankthotrix agardii followed by Aphanizomenon flos-aquae Ralfs ex Bornet \& Flahault (Table 1).

Zooplankton richness ranged between 8 and 14 species in winter and spring respectively (Table $2)$. Rotifers were the most diverse group, and dominated the zooplankton biomass (Fig. 5) (mainly Brachionus angularis Gosse, 1851, Brachionus havanaensis Rousselet, 1911, Keratella tropica Apstein, 1907, Pompholyx complanata Gosse, 1851) and copepods (Microcyclops anceps Richard, 1897). Biomass peaked twice during the study, with the most important peak during the summer (February-March). Total zooplankton biomass was not correlated to any other variable.

Mean total phosphorus (TP) and total nitrogen (TN) were high $(0.42 \mathrm{mgP} / \mathrm{L} \pm 0.05$ and $5.7 \mathrm{mgN} /$ $\mathrm{L} \pm 1.1$, respectively). SRP and $\mathrm{N}_{-} \mathrm{NO}_{3}$ did not show any particular trend, averaging $19.4 \mathrm{~g} \mathrm{P}-\mathrm{PO}_{4}$

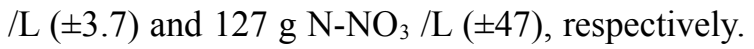
On the other hand, $\mathrm{N}_{-} \mathrm{NH}_{4}$ levels ranged from 48 $830 \mathrm{gN} / \mathrm{L}$ throughout the study and increased by more than 13 times towards the winter. This fact resulted in a slight increase of TN:TP molar ratios and an important increase in DiN:TP molar ratios

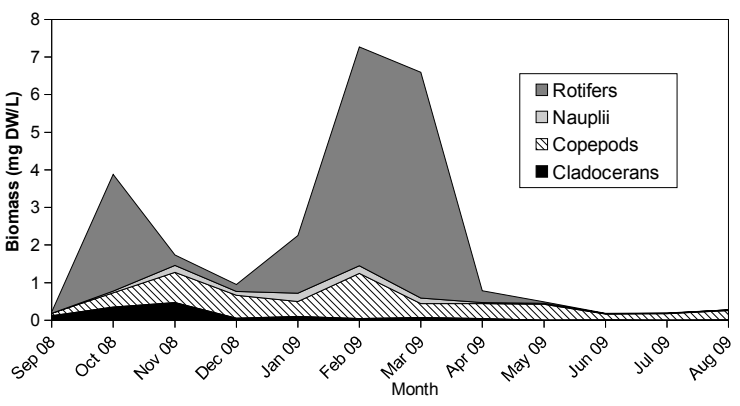

Figura 5. Biomasa (peso seco) de los principales grupos de zooplancton de la laguna Don Tomás, 2008-2009. Redibujado de Echaniz et al. (2012).

Figure 5. Biomass (dry weight) of the main zooplankton groups of Laguna Don Tomás, 2008-2009. Re-drawn from Echaniz et al. (2012).

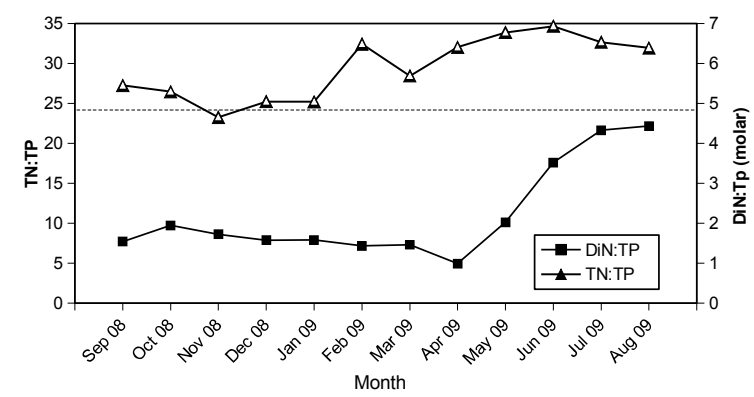

Figura 6. Relaciones molares de $\mathrm{N}$ inorgánico disuelto a $\mathrm{P}$ total (DiN:TP) y $\mathrm{N}$ total a P total (TN:TP) encontrados en la laguna Don Tomás, 2008-2009. La línea punteada indica el límite teórico entre limitación por N y P según Bergstørm (2010).

Figure 6. DiN:TP (black squares) and TN:TP (white triangles) molar ratios found in Laguna Don Tomás, 2008-2009. The dotted line show the theoretical breakpoint between estimated $\mathrm{N}$ limitation and $\mathrm{P}$ limitation using DiN:TP ratios according to Bergstørm (2010).

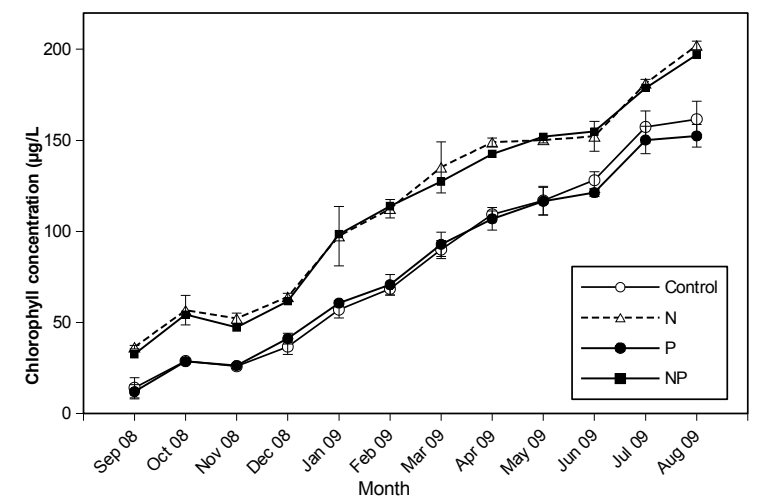

Figura 7. Concentración media final mensual de clorofila $(\mu \mathrm{g} / \mathrm{L})$ en bioensayos de limitación por nutrientes en la comunidad algal de la laguna Don Tomás. Las barras de error indican la desviación estándar. Para todos los meses, las concentraciones de clorofila encontradas con la adición de $\mathrm{N}$ (solo o combinado con $\mathrm{P}$ ) fueron significativamente más altas que las adiciones de $\mathrm{P}$ solo y el control (Tukey, $\mathrm{p}<0.0001$ ).

Figure 7. Mean final chlorophyll concentrations $(\mu \mathrm{g} / \mathrm{L})$ in monthly nutrient limitation bioassays for the phytoplankton community in Don Tomás Lake. Error bars indicate the standard deviation. For all months, chlorophyll concentrations due to $\mathrm{N}$ additions (alone or combined with $\mathrm{P}$ ) were significantly higher than $\mathrm{P}$ additions alone and the control (Tukey, $\mathrm{p}<0.0001$ ). 


\begin{tabular}{|c|c|c|c|c|c|c|c|c|c|c|c|c|}
\hline Especies & Sep-08 & Oct-08 & Nov-08 & Dec-08 & 3 Jan-09 & Feb-09 & Mar-09 & Apr-09 & May-09 & Jun-09 & Jul-09 & Aug-09 \\
\hline \multicolumn{13}{|l|}{ Cyanobacteria } \\
\hline Anabaena aphanizomenoides Forti & 747 & 267 & 587 & 2613 & 160 & 373 & 1600 & 1173 & 213 & 213 & 267 & 267 \\
\hline Anabaena sp. & 107 & 160 & 107 & 640 & 1333 & 533 & 1653 & 2293 & 2667 & 1867 & 1013 & 960 \\
\hline Anabaenopsis arnoldii Aptekar & & & & & 53 & 640 & & 53 & 587 & 107 & 213 & 107 \\
\hline $\begin{array}{l}\text { Aphanizomenon flos-aquae Ralfs ex } \\
\text { Bornet \& Flahault }\end{array}$ & 587 & 1600 & & & 693 & 5920 & 24373 & 40000 & 64747 & 39307 & 18400 & 4000 \\
\hline $\begin{array}{l}\text { Merismopedia tenuissima } \\
\text { Lemmermann }\end{array}$ & & & & & & & & & 107 & & & \\
\hline Microcystis pulverea (H.C.Wood) Forti & 107 & 107 & & 107 & 53 & & 53 & 213 & 53 & 107 & 320 & 53 \\
\hline Oscillatoria proboscidea Gomont & 1973 & 9173 & 10827 & 14347 & 12320 & 16373 & 32000 & 37280 & 9920 & 2027 & 1333 & 1707 \\
\hline $\begin{array}{l}\text { Oscillatoria pseudogeminata } \\
\text { (G.S.Schmid) }\end{array}$ & 373 & 907 & 533 & 800 & 1147 & 427 & 3627 & 3840 & 4213 & 747 & 747 & 1387 \\
\hline Oscillatoria subbrevis Schmidle & 907 & 3040 & 1387 & & 213 & 267 & & & & & 5600 & 6667 \\
\hline Phormidium tenue Gomont & 2293 & 3253 & 2613 & 3413 & 2507 & 4373 & 25280 & 26880 & 14667 & 9333 & 9387 & 5280 \\
\hline $\begin{array}{l}\text { Planktothrix agardhii (Gomont) } \\
\text { Anagnostidis \& Komárek }\end{array}$ & 34080 & 78827 & 57973 & 73707 & 52000 & 49227 & 60373 & 60907 & 63467 & 79467 & 83947 & 64853 \\
\hline Spirulina laxissima G.S.West & 373 & 53 & & & & & 107 & & 267 & 373 & 640 & 1440 \\
\hline Spirulina subsalsa Ørsted ex Gomont & & & & 53 & & & & & & & & \\
\hline Synechococcus sp. & & & & & 1067 & 907 & 3040 & 2613 & 1013 & 480 & 427 & 267 \\
\hline \multicolumn{13}{|l|}{ Chlorophyta } \\
\hline $\begin{array}{l}\text { Actinastrum raphidoides (Reinsch) } \\
\text { Brunnthaler }\end{array}$ & 53 & & & & & 53 & 53 & 267 & 53 & 107 & 160 & 160 \\
\hline Chlamydomonas sp. & 320 & 160 & 213 & 587 & 160 & 533 & 53 & 320 & & 747 & 1600 & 1013 \\
\hline $\begin{array}{l}\text { Closteriopsis acicularis (Chodat) } \\
\text { J.H.Belcher \& Swale }\end{array}$ & & 107 & 53 & 53 & 267 & 1547 & 533 & 213 & 107 & 53 & 160 & 53 \\
\hline Dictyosphaerium elegans Bachmann & & 213 & & 160 & & & 107 & & 107 & 160 & 267 & 160 \\
\hline Golenkinia radiata Chodat & & & 53 & 213 & 107 & 53 & 53 & 320 & & 107 & 160 & 320 \\
\hline $\begin{array}{l}\text { Kirchneriella contorta var. contorta } \\
\text { (Schmidle) Bohlin }\end{array}$ & 320 & 1440 & 320 & 107 & 693 & 107 & & 267 & & 960 & 1280 & 1973 \\
\hline $\begin{array}{l}\text { Kirchneriella obesa (West) West \& } \\
\text { G.S.West }\end{array}$ & 373 & 960 & 587 & 427 & 107 & 53 & 53 & 267 & 320 & 1013 & 1067 & 1760 \\
\hline $\begin{array}{l}\text { Monoraphidium arcuatum (Korshikov) } \\
\text { Hindk }\end{array}$ & 160 & 213 & & 160 & 107 & 53 & 53 & 373 & 267 & 160 & 160 & 1173 \\
\hline $\begin{array}{l}\text { Monoraphidium contortum (Thuret) } \\
\text { Komrkov-Legnerov }\end{array}$ & & & & & & 160 & 53 & 107 & & & & 373 \\
\hline $\begin{array}{l}\text { Monoraphidium griffithii (Berkeley) } \\
\text { Komrkov-Legnerov }\end{array}$ & 160 & 53 & 160 & 267 & 1653 & 1440 & 747 & 213 & 800 & 587 & 640 & 1067 \\
\hline Monoraphidium indicum Hindak & & & & & & 213 & 107 & & 53 & & 213 & 213 \\
\hline $\begin{array}{l}\text { Monoraphidium irregulare (G.M. Smith) } \\
\text { Komrkov Legenerova }\end{array}$ & 53 & 53 & & & & & 427 & 160 & & & 53 & 587 \\
\hline Oocystis lacustris Chodat & 53 & 53 & 53 & 107 & & & & & 107 & & 107 & 107 \\
\hline $\begin{array}{l}\text { Oocystis solitaria Wittrock in Wittrock \& } \\
\text { Nordstedt }\end{array}$ & 53 & & 53 & 213 & 53 & & & 107 & 267 & 747 & 747 & 853 \\
\hline $\begin{array}{l}\text { Pediastrum boryanum (Turpin) } \\
\text { Meneghini }\end{array}$ & & & & & & & & 107 & 160 & 107 & & \\
\hline $\begin{array}{l}\text { Scenedesmus acuminatus (Lagerheim) } \\
\text { Chodat }\end{array}$ & 267 & 587 & & 160 & 267 & 373 & 53 & 160 & 373 & 480 & 533 & 427 \\
\hline $\begin{array}{l}\text { Scenedesmus arcuatus } \\
\text { (Lemmermann) Lemmermann }\end{array}$ & & & & 53 & 53 & 107 & & & & & 53 & 53 \\
\hline $\begin{array}{l}\text { Scenedesmus ecornis (Ehrenber) } \\
\text { Chodat }\end{array}$ & 53 & & & & 320 & & 53 & 320 & & & 107 & 160 \\
\hline Scenedesmus opoliensis P.G. Richter & 53 & & 160 & & & & 160 & 107 & 160 & 53 & 160 & 160 \\
\hline Scenedesmus quadricauda Chodat & 53 & 53 & 213 & 107 & & 53 & & & 53 & 53 & 213 & 267 \\
\hline $\begin{array}{l}\text { Schroederia setigera (Schrder) } \\
\text { Lemmermann }\end{array}$ & 160 & 107 & 160 & 213 & 373 & 320 & 213 & 480 & 427 & 427 & 587 & 907 \\
\hline Tetradron caudatum (Corda) Hansgirg & & & & & & & 53 & 53 & & & & 53 \\
\hline $\begin{array}{l}\text { Tetradron minimum (A. Braun) } \\
\text { Hansgirg }\end{array}$ & 53 & 107 & 53 & 320 & 267 & & 107 & 267 & & 107 & 107 & 213 \\
\hline Tetradron triangulare Korshikov & & & & 53 & 53 & & & 53 & 53 & & & \\
\hline Tetrastrum peterfii Hortobgyi & & & & & & 53 & & & & & 160 & 160 \\
\hline $\begin{array}{l}\text { Tetrastrum staurogeniaeforme } \\
\text { (Schrder) Lemmermann }\end{array}$ & & & & 107 & 53 & & 53 & & & 53 & 53 & 107 \\
\hline \multicolumn{13}{|l|}{ Charophyta } \\
\hline $\begin{array}{l}\text { Staurastrum } s p . \\
\text { Bacillariophyta }\end{array}$ & & & & & & 53 & & & & & & \\
\hline $\begin{array}{l}\text { Aulacoseira granulata (Ehrenberg) } \\
\text { Simonsen }\end{array}$ & & 107 & 533 & 160 & 1067 & 747 & 693 & 320 & 107 & & 213 & 533 \\
\hline Cyclotella meneghiniana Ktzing & & & 267 & 267 & 480 & 533 & 533 & 693 & 213 & 267 & 480 & 800 \\
\hline Fragilaria sp. & 107 & & 320 & 747 & 1333 & 533 & 1760 & 693 & 1440 & 373 & 213 & 427 \\
\hline $\begin{array}{l}\text { Euglenozoa } \\
\text { Euglena variabilis G.A.Klebs } \\
\text { Miozoa }\end{array}$ & & 427 & & 267 & 107 & 480 & 480 & 160 & 53 & 1280 & 1173 & 640 \\
\hline Peridinium sp. & 107 & 320 & & 107 & 107 & 160 & 107 & & & & 267 & 107 \\
\hline Cryptophyta & & & & & & & & & & & & \\
\hline Cryptomonas ovata Skuja & & 107 & & & & 53 & 107 & 53 & 53 & 267 & 53 & 267 \\
\hline
\end{tabular}

Tabla 1. Abundancia de especies de fitoplancton (organismos por mililitro) encontradas en la laguna Don Tomás durante el estudio.

Table 1. Phytoplankton species abundance (organisms per $\mathrm{mL}$ ) found in Don Tomás lake during the study. 


\begin{tabular}{|c|c|c|c|c|c|c|c|c|c|c|c|c|}
\hline Especies & Sep-08 & Oct-08 & Nov-08 & Dec-08 & Jan-09 & Feb-09 & Mar-09 & Apr-09 & May-09 & Jun-09 & Jul-09 & Aug-09 \\
\hline \multicolumn{13}{|l|}{ Cladocerans } \\
\hline Bosmina huaronensis Delachaux, 1918 & 138 & 605 & 817 & 107 & 257 & 117 & 15 & 110 & 10 & 8 & 6 & 10 \\
\hline Diaphanosoma birgei Korinek, 1981 & & & & & & 22 & 18 & & & & & \\
\hline \multicolumn{13}{|l|}{ Copepods } \\
\hline $\begin{array}{l}\text { Acanthocyclops robustus (G.O. Sars, } \\
1863)^{*}\end{array}$ & & & & & & & & & 1 & 6 & & \\
\hline Microcyclops anceps (Richard, 1897)* & 15 & 180 & 361 & 393 & 412 & 733 & 445 & 370 & 157 & 39 & 72 & 66 \\
\hline $\begin{array}{l}\text { Nauplii } \\
\text { Rotifers }\end{array}$ & 35 & 289 & 1612 & 627 & 1467 & 1193 & 802 & 213 & 97 & 12 & 84 & 54 \\
\hline Anuraeopsis fissa (Gosse, 1851) & & 30 & & & & & 20 & & & & & 6 \\
\hline Brachionus plicatilis Mller, 1786 & 9 & 11 & & & & 7 & & 17 & & & & \\
\hline Brachionus angularis Gosse, 1851 & & 255 & 3 & & 77 & 213 & 3500 & & 1 & 4 & & \\
\hline $\begin{array}{l}\text { Brachionus havanaensis Rousselet, } \\
1913\end{array}$ & & 45 & 1087 & 13 & 157 & 8440 & 7200 & 7 & 2 & 7 & 6 & \\
\hline Brachionus dimidiatus Bryce, 1931 & & 10 & & & & & & 3 & & & & \\
\hline Brachionus calyciflorus (Pallas, 1766) & 2 & & & 4 & 57 & 80 & 165 & & & & & \\
\hline Colurella sp. & & 5 & & & & & & & & & & \\
\hline Filinia longiseta (Ehrenberg, 1834) & & 5 & & & & & & & & & & \\
\hline $\begin{array}{l}\text { Hexarthra intermedia (Wiszniewski, } \\
\text { 1929) }\end{array}$ & & & & 103 & 733 & 477 & 205 & & & & & \\
\hline Keratella cochlearis (Gosse, 1851) & 158 & 1370 & 813 & & & & & & & & & \\
\hline Keratella tropica (Apstein, 1907) & 38 & 11520 & 760 & 517 & 1913 & 5020 & 2750 & 2377 & 520 & 20 & 10 & 4 \\
\hline Lecane sp. & 3 & & & & & & & & & & & \\
\hline Polyarthra dolichoptera Idelson, 1925 & 78 & 35 & 83 & 17 & 197 & 187 & 170 & 107 & 17 & 4 & 24 & 50 \\
\hline Pompholyx complanata Gosse, 1851 & 93 & 6600 & 97 & 37 & 647 & 67 & 800 & 1397 & 3 & 44 & 38 & 4 \\
\hline Synchaeta sp. & & & & & & 10 & 20 & & & & 10 & 2 \\
\hline
\end{tabular}

Tabla 2. Abundancia de especies de zooplancton (individuos por mililitro) encontradas en la laguna Don Tomás durante el estudio. Los asteriscos indican que el número incluye adultos y estadios copepoditos.

Table 2. Zooplankton species abundance (individuals per liter) found in Don Tomás lake during the study. The asterisk indicate that the number comprises adults and copepodite stages.

(Fig. 6). DiN:TP molar ratios were always less than 4.5 during the study.

The phytoplankton community was N-limited all year long (Fig. 7). Bioassays with $\mathrm{N}$ additions (N or $\mathrm{N}$ plus $\mathrm{P}$ ) always had a higher chlorophyll response than the control or $\mathrm{P}$ treatments (ANOVA: $\mathrm{F}_{2,33}=91.35 ; \mathrm{p}<0.0001$ ). $\mathrm{N}$ treatments averaged $64 \%$ of controls, and $\mathrm{N}+\mathrm{P}$ treatments were $58 \%$ of controls whereas $\mathrm{P}$ treatments averaged $-1 \%$ of controls.

\section{Discussion}

Don Tomás lake frequently had summer chlorophyll peaks (Echaniz et al. 2008). The existence of an important, non-frequent, autumn chlorophyll peak (Fig. 2) in 2009 gave us the chance to analyze the factors that may have induced this phenomenon. There are two main factors that could have influenced this late in the season peak: reduced zooplankton grazing pressure (Top-Down effect) and nutrient/light stimulus (Bottom-Up effect).

Regarding the first factor, it is important to note that Don Tomás zooplankton taxonomic composition has dramatically changed in the last 13 years due to predation on large zooplankton by silversides (Odontesthes bonariensis). When the current zooplankton richness was compared to that from more than a decade ago (Echaniz et al. 2012), we observed that 7 cladocerans and 2 copepod species were lost (with Daphnia obtusa Kurz, 1874 and Daphnia spinulata Biraben, 1917, among the largest) and 8 small-sized new rotifer species were found by 2008-9 (B. dimidiatus, $B$. calyciflorus, $K$. tropica, H. intermedia, $P$. complanata, Lecane sp. Nitzsch, 1827, A. fissa, Synchaeta sp. Ehrenberg, 1832). Since microzooplankton have lower grazing efficiencies than macrozooplankton, a reduction in mean water transparency was consequently observed $(0.24 \mathrm{~m}$ in 1995-96 to $0.15 \mathrm{~m}$ in 2006) (Echaniz et al. 2008). During the present study, Don Tomás zooplankton biomass was dominated by rotifers and small-sized copepods (Fig. 5) which have low grazing efficiencies. Particularly, during May and June (fall/winter) when the chlorophyll peak was observed, there was a notorious lack of grazers (Fig. 5) $(0.2-0.5 \mathrm{mgDW} / \mathrm{L})$ that could have released the grazing pressure on the algal community even more. At this very same time, the phytoplankton community was dominated by cyanobacteria (94.3-96.9\% of the whole algal community biovolume) (Fig. 4). Cyanobacteria is considered a low quality food resource group for zooplankton and produces a variety of toxins which induce limitations to the survival, growth and reproduction of zooplanktonic organisms (Lampert 1987, Ghadouani et al. 2003). Thus, we assumed that the zooplankton groups present when chlorophyll 
peaked, did not exert a strong grazing pressure on the algal community in order to control its biomass as was already observed for another hypereutrophic Pampean shallow lake by Diovisalvi et al. (2010).

The second factor that could have affected this late in the season chlorophyll peak in 2008-2009 is a direct response to a nutrient/light stimulus. Don Tomás nutrient concentrations were high, and consequently affected the phytoplankton community nutrient limitation. As predicted by Bergström (2010), and corroborated by our bioassays, Don Tomás's phytoplankton community was always N-limited, as DiN:TP molar ratios were always lower than 4.9 (Fig. 7). This corroborates the findings by Halstvedt et al. (2007) who reported N-limitation in Planktothrix rubescens (De Candolle ex Gomont) Anagnostidis \& Komárek and $P$. agardhii populations in enrichment experiments, and that Don Tomás Lake cyanobacteria community was dominated by $P$. agardhii. In Don Tomás, we also observed that the response to $\mathrm{N}$ additions was significantly inversely correlated to $\operatorname{DiN}\left(R_{s}=-0.602 ; p=0.038\right)$. Nevertheless, chlorophyll concentrations were not correlated to $\operatorname{DiN}\left(\mathrm{R}_{\mathrm{s}}=0.347 ; \mathrm{p}=0.270\right)$ indicating that nitrogen availability would not be enough to regulate the chlorophyll increase during the autumn.

In Don Tomás, phytoplankton biomass and chlorophyll-a concentration peaked in autumn (Fig. 2), but these two peaks were approximately 2-months apart. When reviewing this phenomenon elsewhere, Kasprzak et al. (2008) indicated that this decoupling was mainly affected by seasonal changes in the algal community or changes in the trophic status. In Don Tomás, these two factors can be discarded as the phytoplankton community was always dominated by the cyanobacteria (Fig. 4) and nutrient levels were high enough (Fig. 6) to classify this lake as permanently hypereutrophic. On the other hand, Felip \& Catalán (2000) found that a Spanish oligotrophic lake had a biovolume peak a month earlier than chlorophyll-a concentration. These authors suggested that a change in the per-unit cell chlorophyll concentration as a response to winter light-limitation was the main factor affecting this decoupling. This situation is the most compatible one with Don Tomás' as we found that Don Tomás lake has a mean transparency of $0.14 \mathrm{~m}$, meaning an estimated euphotic depth of $0.46 \mathrm{~m}$ (Kalff 2003), approximately of the mean depth registered at the beginning of the study. Thus, approximately of the water column within Don Tomás Lake might be under strong light limitation (which could have been alleviated by the polymictic nature of this lake). Light limitation is common in turbid, shallow lakes of Argentinas' pampas (Quirós \& Drago 1999, Torremorell et al. 2007, 2009, Diovisalvi et al. 2015). In addition to the low water transparency, it is necessary to consider the natural light availability reduction during autumn and winter. In order to cope with light limitation, algal cells might increase their pigment concentration as a physiological adaptation to low irradiance, consequently reducing the $\mathrm{C}: \mathrm{Chl}$ ratio (Cullen 1982). If this is the case here, some changes in $\mathrm{C}$ :Chl ratios would be expected in Don Tomás. Although we did not measure $\mathrm{C}$ content in phytoplankton directly, we estimated it at $10 \%$ of algal biovolume (Peters and Downing 1984). The mean C:Chl ratio of 78 indicated a cyanobacteria dominated lake as suggested by Yacobi \& Zohari (2010). This ratio also had lower values during autumn and winter (Fig. 2). According to Cullen (1982) these low values indicate higher chlorophyll concentrations in phytoplankton, typical of periods with low solar radiation (typical of fall/ winter months) and high nutrient concentrations (Taylor et al. 1997) as observed in figure 6.

As mentioned previously, Don Tomás lake has shown both, phytoplankton successions with typical summer chlorophyll peaks (Echaniz et al. 2008) as well as autumn-winter peaks (Echaniz et al. 2012; this study). Winder \& Cloern (2010) found that phytoplankton biomass peaks may happen regularly (every 6 or 12 months) or irregularly. Irregular occurrence was common in hypereutrophic systems dominated by cyanobacteria, low grazing pressure and short-term climatic variations where Don Tomás Lake would easily fit. Nevertheless, in order to understand the true dynamics of this shallow, urban lake, it will be necessary to include climatic variables in future long-term monitoring since precipitation variability on the west side of the Pampa's plains (Viglizzo 2011) seems to cause important variations in some of the lake's parameters (e.g., depth, salinity, light and nutrient availability).

\section{Conclusion}

Don Tomás Lake had an important 2009 fall 
chlorophyll peak due to the lack of large grazers, which together with the dominance of the nongrazeable $P$. agardhii, probably weakened topdown processes. On the other hand, the presence of bottom-up processes fueled by high nutrients in this system might have stimulated algal growth. The chlorophyll peak occurred later than the biomass peak as phytoplankton cells increased their chlorophyll content in spite of algal biomass (biovolume) probably due to a reduction of solar irradiance and low water transparency. A longterm monitoring would be needed in order to analyze if unusual blooms are affected by climatic variations.

\section{Acknowledegments}

This research was funded by Universidad Nacional de La Pampa, project 202 and Préstamo BID PRH-PICT 00301. Daniel Sierra, Claudia Monteira, and "Quito" Baumgaertner (Municipal Canoeing School personnel) helped us with field logistics.

\section{References}

APHA (American Public Health Association). 1992. Standard Methods for the Examination of Water and Wastewater. Washington DC, USA: American Public Health Association, American Water Works Association, and Water Environment Federation.

Bergström AK. 2010. The use of TN:TP and DiN:TP as indicators for phytoplankton nutrient limitation in oligotrophic lakes affected by $\mathrm{N}$ deposition. Aquatic Sciences 72: 277-281.

Bourrely P. 1985. Les algue d'eau douce. Initiation à la systématique. Tome III: Les Algues bleues et rouges. Les Eugléniens, Peridiniens et Cryptomonadines. París: Société Nouvelle des Éditions Boubée.

Carlson RE. 1977. A trophic state index for lakes. Limnology and Oceanography 22 (2): 361-369.

Cullen JJ. 1982. The deep chlorophyll maximum: comparing vertical profiles of chlorophyll a. Canadian Journal of Fisheries and Aquatic Sciences 39: 291803.

Diovisalvi N, Berasain G, Unrein F, Colauti D, Fermani P, Llames ME, ... \& Zagarese H. 2010. Chascomús: estructura y funcionamiento de una laguna pampeana turbia. Ecología Austral 20 (2): 115-127.

Diovisalvi N, Bohn VY, Piccolo MC, Perillo GME, Baigún C \& Zagarese H. 2015. Shallow lakes from the Central Plains of Argentina: an overview and worldwide comparative analysis of their basic limnological features. Hydrobiologia 752: 5-20.

Ebina J, Tsuyoshi T \& Totoso S. 1983. Simultaneous de- termination of total nitrogen and total phosphorus in water using peroxodisulfate oxidation. Water Research 17 (12): 1721-1726.

Echaniz SA, Vignatti MA \& Bunino PC. 2008. El zooplancton de un lago somero hipereutrófico de la región central de Argentina: cambios después de una década. Biota Neotrópica 8 (4): 63-71.

Echaniz SA, Vignatti MA, Pilati A \& Kissner S. 2012. Cambios en la diversidad y variación interanual de la abundancia del zooplancton de un lago somero urbano de La Pampa. Biología Acuática 27: 97-112.

Echaniz SA, Cabrera GC, Aliaga PL, \& Vignatti AM. 2013. Variations in zooplankton and limnological parameters in a saline lake of La Pampa, central Argentina, during an annual cycle. International Journal of Ecosystem 3 (4): 72-81.

Echaniz SA, Vignatti MA, Cabrera GC, Capecce C \& Treffinger-Cienfuegos MS. 2016. Comparación de los parámetros limnológicos y el zooplancton de un lago somero salino de La Pampa (Argentina) durante dos ciclos anuales de condiciones diferentes. Biología Acuática 31: 28-39.

Felip M \& Catalán J. 2000. The relationship between phytoplankton biovolume and chlorophyll in a deep oligotrophic lake: decoupling in their spatial and temporal maxima. Journal of Plankton Research 22 (1): 91-105.

Ghadouani A, Pinel-Alloul B \& Prepas EE. 2003. Effects of experimentally induced cyanobacterial blooms on crustacean zooplankton communities. Freshwater Biology 48: 363-381.

Halstvedt CB, Rohrlack T, Andersen T, Skilberg O \& Edvarsen B. 2007. Seasonal dynamics and depth distribution of Planktothrix spp. in Lake Steinsfjorden (Norway) related to environmental factors. Journal of Plankton Research 29 (5): 471-482.

Hillebrand $H$, Dürselen $C D$, Kirschtel D, Pollingher U \& Zohary T. 1999. Biovolume calculation for pelagic and benthic microalgae. Journal of Phycology 35: 403-424.

Izaguirre I, Sánchez ML, Schiaffino MR, O'Farrel I, Huber P, Ferrer N, ... \& Mancini M. 2015. Which environmental factors trigger the dominance of phytoplankton species across a moisture gradient of shallow lakes? Hydrobiologia 752: 47-64.

Kalff J. 2003. Limnology: inland water ecosystems. New Jersey: Prentice Hall.

Kasprzak P, Padisák J, Koschela R, Krientiza L \& Gervaisa F. 2008. Chlorophyll a concentration across a trophic gradient of lakes: An estimator of phytoplankton biomass? Limnologica 38: 327-338.

Komárek J \& Anagnostidis K. 1999. Cyanoprokaryota part I: Chroococcales. Süßwasserflora von Mitteleuropa 19/1. Heidelberg: Spektrum Akademischer Verlag.

Komárek J \& Anagnostidis K. 2007. Cyanoprokaryota part 2: Oscillatoriales. Süßwasserflora von Mitteleuropa 19/2. Heidelberg: Spektrum Akademischer Verlag.

Komárek J. 2013. Cyanoprokaryota. 3. Heterocytous genera. Süswasserflora von Mitteleuropa/Freshwater flora of Central Europe. Heidelberg: Springer 
Spektrum Berlin.

Lampert W. 1987. Laboratory studies on zooplanktoncyanobacteria interactions. New Zealand Journal of Marine and Freshwater Research 21 (3): 483-490.

Lampert W \& Sommer U. 2007. Limnoecology. 2nd Edition. New York: Oxford University Press.

Marcarelli AM, Wurtsbaugh WA \& Griset O. 2006. Salinity controls phytoplankton response to nutrient enrichment in the Great Salt Lake, Utah, USA. Canadian Journal of Fisheries and Aquatic Sciences. 63: 2236-2248.

Morello J, Matteucci S, Rodríguez A \& Silva M. 2012. Ecorregiones y complejos ecosistémicos argentinos. Buenos Aires: Orientación Gráfica Editores.

Moustaka-Gouni M, Michaloudi E \& Sommer U. 2014. Modifying the PEG model for Mediterranean lakes no biological winter and strong fish predation. Freshwater Biology 59: 1136-1144.

Peters RH \& Downing JA. 1984. Empirical analysis of zooplankton filtering and feeding rates. Limnology and Oceanography 29 (4): 763-784.

Prescott GW. 1951. Algae of the Western Great Lakes Area: Exclusive of desmids and diatoms. Bulletin 31. Bloomfield Hills MI, USA: Cranbrook Institute of Science.

Quirós R \& Drago E. 1999. The environmental state of Argentinean lakes: an overview. Lakes \& reservoirs: Research and Management 4: 55-64.

Quirós R, Rennella AM, Boveri MV, Rosso JJ \& Sosnovsky A. 2002. Factores que afectan la estructura y funcionamiento de las lagunas pampeanas. Ecología Austral 12: 175-185.

Sommer U, Gliwicz ZM, Lampert W \& Duncan A. 1986.
The PEG-model of seasonal sucesión of planktonic events in fresh waters. Archiv für Hydrobiologie 106: 433-471.

Taylor AH, Geider JR \& Gilbert FJH. 1997. Seasonal and latitudinal dependencies of phytoplankton carbon-to-chlorophyll a ratios: results of a modelling study. Marine Ecology Progress Series 152: 51-66.

Torremorell A, Bustingorry J, Escaray R, \& Zagarese HE. 2007. Seasonal dynamics of a large, shallow lake, laguna Chascomús: the role of light limitation and other physical variables. Limnologica 37: 100108.

Torremorell A, Llames ME, Pérez GL, Escaray R, Bustingorry $\mathrm{J} \&$ Zagarese H. 2009. Annual patterns of phytoplankton density and primary production in a large, shallow lake: the central role of light. Freshwater Biology 54: 437-449.

Viglizzo EF. 2011. El agro, el clima y el agua en La Pampa semiárida: revisando paradigmas. Tomo LXIV. Jornada "Condiciones para el Desarrollo de Producciones Agrícola-Ganaderas en el S.O. Bonaerense". Bahía Blanca, Argentina: Academia Nacional de Agronomía y Veterinaria, pp 251-267.

Vignatti AM, Cabrera GC, Canosa M \& Echaniz SA. 2017. Environmental and zooplankton parameter changes during the drying of a saline shallow temporary lake in central Argentina. Universitas Scientarium 22 (3): 177-200.

Winder M \& Cloern JE. 2010. The annual cycles of phytoplankton biomass. Philosophical Transaction of the Royal Society B. 365: 3215-3226.

Yacobi YZ \& Zohary T. 2010. Carbon:chlorophyll a ratio, assimilation numbers and turnover times of Lake Kinneret phytoplankton. Hydrobiologia 639: 185-196. 